\title{
Granulomatous Hepatitis: A Rare Primary Manifestation of Disseminated Histoplasmosis in a Renal Transplant Recipient
}

\author{
Jiten P. Kothadia ${ }^{1}$, Vyshnavi Kone ${ }^{1}$, Shah Giashuddin ${ }^{2}$
}

1) Nazih Zuhdi Transplant Institute, INTEGRIS Baptist Medical Center, Oklahoma City, OK; 2) Department of Pathology and Laboratory Medicine, New York-Presbyterian Brooklyn Methodist Hospital, Brooklyn, NY, USA
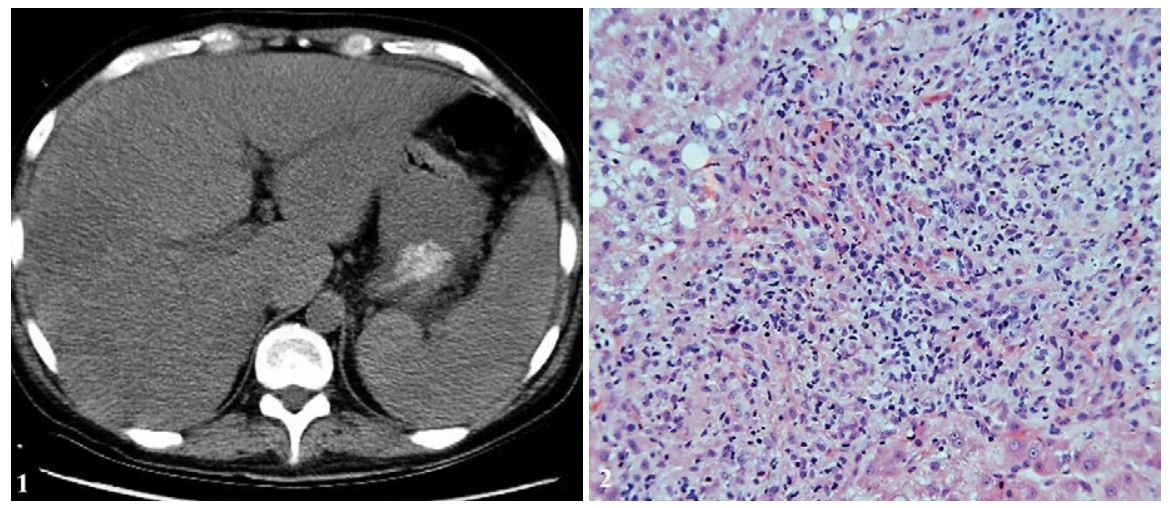

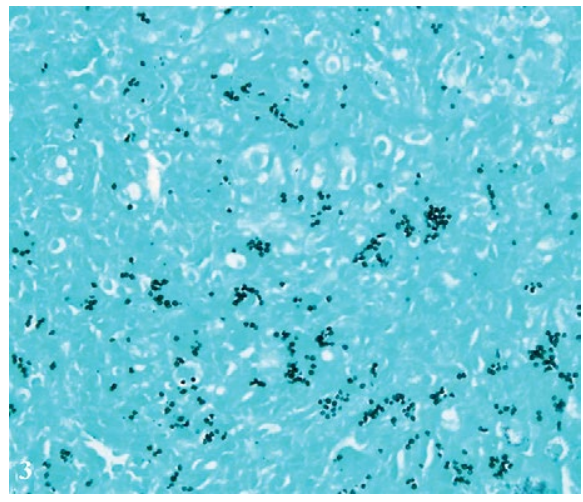

A 41-year-old white male with past medical history significant for renal transplantation in 2007, was admitted with malaise, fever and worsening jaundice. Four days before admission he underwent an uneventful laparoscopic cholecystectomy with liver biopsy. Liver biopsy revealed moderate to severe mixed portal tract infiltration with unknown etiology.

Physical examination was benign except the cervical lymphadenopathy. His laboratory tests showed anemia, leukopenia, and thrombocytopenia. Liver function tests were altered: aspartate aminotransferase 70 (3-50) IU/L, alanine aminotransferase 68 (3-60) IU/L, alkaline phosphatase 1351 (20-125) IU/L, total bilirubin $10.2(0.2-1.3) \mathrm{mg} / \mathrm{dL}$. Tests for viral hepatitis, autoimmune markers, serum rapid plasma reagin (RPR), QuantiFERON-TB Gold, Ebstein-Barr virus (EBV), and cytomegalic virus (CMV) were all negative. Blood and urine cultures were negative. He was tested positive for human immunodeficiency virus (HIV). Computed tomography (CT) of the abdomen showed heterogeneous lesions in the right hepatic lobe suspicious for infection with mesenteric and retroperitoneal lymphadenopathy (Fig. 1). Upon re-reviewing, liver biopsy revealed fungal granulomatous inflammation (Fig. 2, areas of non-necrotizing granulomatous inflammation without any giant cells but many histiocytes in the background). Gomori Methenamine Silver (GMS) staining highlighted round to ovoid, narrow based budding yeasts, suggestive of histoplasmosis (Fig. 3). Quantitative MVista Histoplasma antigen enzyme immunoassay test confirmed the findings with Histoplasma antigen titers $>25 \mathrm{ng} / \mathrm{mL}$. The patient was initiated with itraconazole and highly active antiretroviral (HART) therapy for further management. The patient succumbed to severe infection with multiorgan system failure within three weeks of admission.

The dimorphic fungus Histoplasma capsulatum causes the most common primary mycosis in the United States, which can often progress to disseminated histoplasmosis in immunocompromised patients $[1,2]$. Liver involvement because of disseminated histoplasmosis is common. However, liver histoplasmosis as an initial sign of histoplasmosis, without lung involvement is very rare [1-3]. A high index of suspicion for histoplasmosis is required especially for renal transplant recipients. Early diagnosis is crucial, as treatment delays is associated with increasing morbidity and mortality.

Corresponding author: Jiten P. Kothadia, kothadia.jiten@gmail.com

Conflicts of interest: None to declare.

\section{REFERENCES}

1. Rihana NA, Kandula M, Velez A, Dahal K, O’Neill EB. Histoplasmosis presenting as granulomatous hepatitis: case report and review of the literature. Case Rep Med 2014;2014:879535. doi:10.1155/2014/879535

2. Lo MM, Mo JQ, Dixon BP, Czech KA. Disseminated histoplasmosis associated with hemophagocytic lymphohistiocytosis in kidney transplant recipients. Am J Transplant 2010;10:687-691. doi:10.1111 /j.1600-6143.2009.02969

3. Zoutman DE, Ralph ED, Frei JV. Granulomatous hepatitis and fever of unknown origin: An 11-year experience of 23 cases with three years' follow-up. J Clin Gastroenterol 1991;13: 69-75. 